Introduction Diffuse idiopathic pulmonary neuroendocrine cell hyperplasia (DIPNECH) is a rare condition characterised by a generalised proliferation of pulmonary neuroendocrine cells within the respiratory epithelium. Current literature is limited, in particular little is known of its affects on pulmonary function both at the time of diagnosis and prospectively, though it is recognised to cause small airway obstruction.

Objective The aim of this study was to characterise pulmonary function, both at baseline and to also define the change in pulmonary function over time in patients with DIPNECH.

Methods Retrospective analysis of pulmonary function data for patients with a histological diagnosis of DIPNECH was performed. At baseline, pulmonary function was characterised as either obstructive, small airways obstruction, restrictive, mixed (obstructive and restrictive) or normal. Baseline gas transfer (DLCO) and lung volume data was also described. FEV1 was used as the main measure of pulmonary function, and simple linear regressions were created for patients with longitudinal data. This then allowed basic statistical analysis of the change in FEV1 compared to the predicted change.

Results 17 patients ( $82 \%$ female), with a mean age of 59, were included. All had pulmonary function data at baseline and $9(53 \%)$ had prospective data. Baseline pulmonary function was predominantly obstructive in nature with $6(35 \%)$ having classical obstruction, and 7 (41\%) small airways obstruction alone with a normal FEV1/FVC ratio, the remaining 4 having either normal $(n=3,23 \%)$ or mixed $(n=1,6 \%)$ physiology. The mean FEV1 at baseline was $81.6 \%$, and a statistically significant difference was present between mean measured and predicted FEV1 values for the cohort $(p=0.02)$. Mean DLCO $(n=13)$ was mildly decreased at $84.6 \%$ predicted however corrected to normal with volume. Lung volume data $(n=8)$ where available was normal, except in two patients $(12 \%)$ who had significantly increased residual volume. Patients with longitudinal data $(\mathrm{n}=9,53 \%)$ predominantly showed a stable pattern of obstruction with minimal decline. Two patients (12\%) did have a significantly increased decline compared to predicted values.

Conclusion Patients with DIPNECH typically have a stable degree of fixed obstruction, however exceptions to this will be seen in patients with a more progressive disease.

\section{P142 HYPOXIC CHALLENGE TEST (HCT) FOR IN-FLIGHT OXYGEN ASSESSMENTS CAN BE AVOIDED IN PATIENTS WITH LUNG DISEASE AND LOW RESTING PAO2}

R Peat, F Frost, K Waldron, J Furlong, D Russell, D Wat. Liverpool Heart and Chest Hospital, Liverpool, UK

\subsection{6/thoraxjnl-2017-210983.284}

Introduction Hypoxic challenge test consisting of breathing $15 \% \mathrm{FiO} 2$ for $20 \mathrm{~min}$ with blood gas measurements is recommended by BTS guidelines for the assessment of the requirement for in-flight oxygen. FEV1 and SpO2 have been demonstrated to be poor predictors of desaturation with no established reliable upper limit of $\mathrm{PaO} 2$, above which patients will not desaturate. We investigated whether there were lowerlimit thresholds, beyond which oxygen is always required and hence HCT can be avoided.

Methods Retrospective analysis of all hypoxic challenge tests conducted at our centre between 2010 and 2017 was undertaken. Baseline demographics, diagnosis and contemporaneous lung function data was recorded. HCT was performed as per BTS guidance and included baseline resting blood gas followed by a repeat after $20 \mathrm{~min}$ inspiring $15 \% \mathrm{FiO} 2$. If $\mathrm{PaO} 2$ was $<6.65 \mathrm{kPa}$ or $\mathrm{SpO} 2<85 \%, 2 \mathrm{~L}$ oxygen via nasal cannulae was applied and a repeat blood gas performed to confirm $\mathrm{PaO} 2 \geq 6.65 \mathrm{kPa}$.

Results HCT was performed on 170 occasions during the study period. COPD was the underlying diagnosis in 110 (64.7\%) of tests, ILD in 40 (23.5\%) and CF (13, 11.8\%). Average age (median [range]) was 67.5 years [49.1-83.8] COPD, 67 [52.3-83.3]ILD, 32.5 [19.1-66.8]CF. Lung function (FEV1\%pred) was 49.7[21-115]COPD, 71.6 [31-124]ILD, 36.5[23-65]CF. Following HCT, in-flight oxygen was recommended in $99(58.2 \%)$ patients all of whom were recommended $21 / \mathrm{min}$. A threshold of $<7.55 \mathrm{Kpa}$ on resting blood gas was $100 \%$ predictive for requirement of in-flight oxygen and a threshold of $<8 \mathrm{kPa}$ was $97.9 \%$ predictive. Incorporating the $<7.55 \mathrm{kPa}$ and $<8 \mathrm{kPa}$ thresholds into clinical practice by proceeding straight to 21 oxygen could negate the need for HCT in $20.6 \%$ and $43.9 \%$ of cases respectively.

Conclusion HCT is a useful tool for assessing the need for inflight oxygen in lung diseases but is a resource heavy test and requires multiple blood samples taken from patients. Our data suggests that there are lower-limit thresholds for resting $\mathrm{PaO} 2$ beyond which HCT can be avoided in a significant proportion of patients.

\section{P143 USING BIG DATA TO INVESTIGATE PHYSIOLOGY: RETENTION OF CO2 DOES NOT IMPACT THE OXYGEN- HAEMOGLOBIN DISSOCIATION CURVE OF CRITICALLY ILL ADULTS}

${ }^{1} \mathrm{~N}$ Rosculet, ${ }^{1} \mathrm{R}$ Samata, ${ }^{1} \mathrm{~A}$ Dixit, ${ }^{2} \mathrm{~S}$ Harris, ${ }^{2} \mathrm{NS}$ MacCallum, ${ }^{2} \mathrm{DA}$ Brearley, ${ }^{3} \mathrm{PJ}$ Watkinson, ${ }^{4} \mathrm{~A}$ Jones, ${ }^{5} \mathrm{~S}$ Ashworth, ${ }^{4} \mathrm{R}$ Beale, ${ }^{5} \mathrm{SJ}$ Brett, ${ }^{3} \mathrm{JD}$ Young, ${ }^{2} \mathrm{M}$ Singer, ${ }^{1} \mathrm{C}$ Summers, ${ }^{1} \mathrm{~A}$ Ercole. ${ }^{1}$ Department of Medicine, University of Cambridge School of Clinical Medicine, Cambridge, UK; ${ }^{2}$ Bloomsbury Institute of Intensive Care Medicine, University College London, London, UK; ${ }^{3}$ Nuffield Department of Clinical Neurosciences, University of Oxford, Oxford, UK; ${ }^{4}$ Department of Intensive Care Medicine, Guy's and St Thomas' Hospital NHS Foundation Trust, London, UK; ${ }^{5}$ Centre for Perioperative Medicine and Critical Care Research, Imperial College Healthcare NHS Trust, London, UK

\subsection{6/thoraxjnl-2017-210983.285}

Introduction Since its initial description in 1904, the oxygenhaemoglobin dissociation curve (ODC) has been well described under physiological conditions. ${ }^{1,2}$ However, the impact of pathology has been less well characterised, with most data arising from small clinical studies of anaesthetized adults/ patients $(<100$ subjects), or experimentally-induced hypoxaemia/hypercapnia. Routinely collected clinical data, including arterial blood gas analyses, are now available from many thousands of critically ill patients. We sought to investigate the impact of $\mathrm{pCO}_{2}$ on the ODC of critically ill adults, and hypothesised that $\mathrm{pCO}_{2}$ would not significantly alter the relationship between $\mathrm{pO}_{2}$ and haemoglobin saturation.

Methods Data was extracted from the National Institute for Health Research Critical Care Health Informatics Collaborative (NIHR ccHIC). Statistical analysis was undertaken on 399000 blood gases from 13942 patients, using R version 3.4.0. After data cleaning, the predicted oxygen saturation for each arterial blood gas sample was calculated using both the Severinghaus ${ }^{1}$ and Dash, Kroman and Bassingthwaighte ${ }^{2}$ equations. Non-linear regression modelling was undertaken to construct ODCs based on both the predicted and observed data, 
Oxygen-Haemoglobin Dissociation Curve

(by $\mathrm{PaCO}_{2}$ levels)

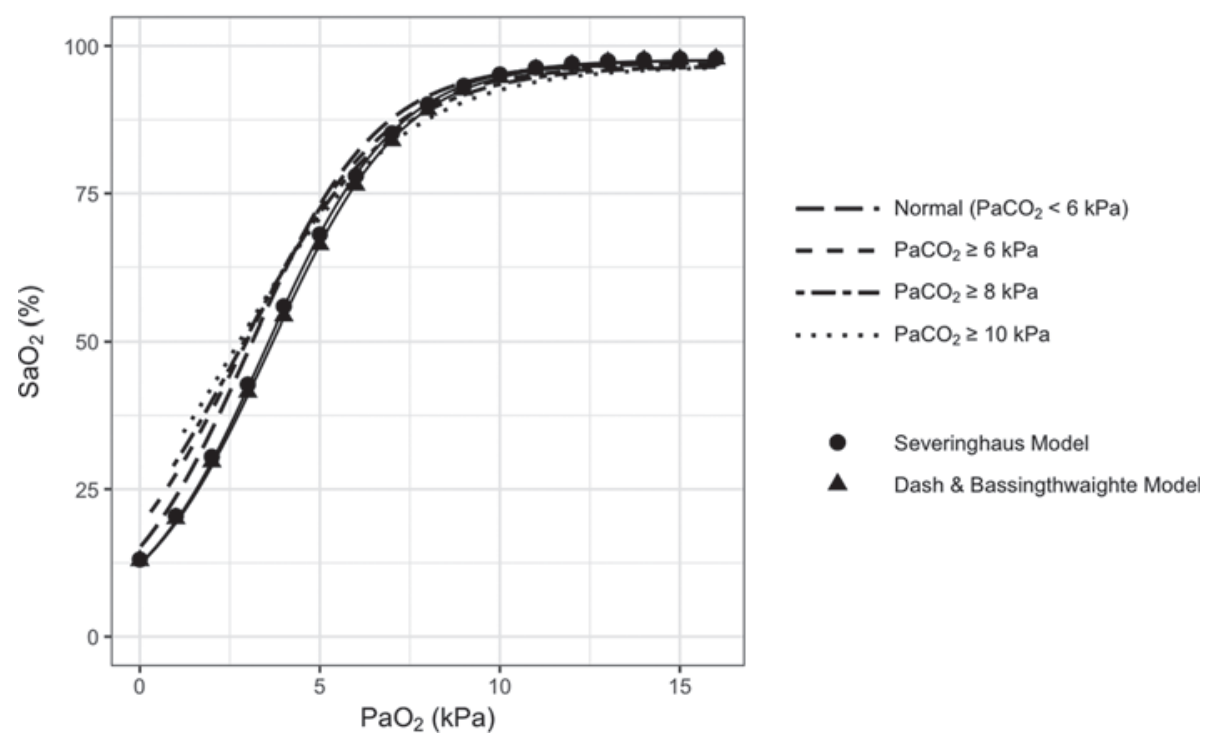

Abstract P143 Figure 1 The observed oxygen dissociation curve of critically ill patients with varying levels of hypercapnia.

to allow comparison. Observed data was stratified into strata based on $\mathrm{pCO} 2$ to investigate the influence of hypercapnia on the ODC.

Results No clinically significant impact of $\mathrm{pCO} 2$ on the relationship between $\mathrm{pO}_{2}$ and oxygen saturation was observed in samples obtained from critically ill adults (mean difference $0.35 \mathrm{kPa}(\mathrm{SD}=0.2 \mathrm{kPa})$ for a given oxygen saturation). Interestingly, we did not observe "right shift" of the ODC in response to elevated arterial $\mathrm{pCO} 2$, and there was no impact of either acute $\left(\mathrm{HCO}_{3}<28 \mathrm{mmol} / \mathrm{L}\right)$ or chronic $\left(\mathrm{HCO}_{3} \geq 28 \mathrm{mmol} / \mathrm{L}\right)$ hypercapnia on the relationship between haemoglobin saturation and $\mathrm{pO} 2$.

Conclusions These data suggest that the relationship between haemoglobin saturation and $\mathrm{pO} 2$ described by data from small scale studies may not reflect physiology observed in critically ill adults, and further that the right shift of the ODC reported in experimental hypercapnia, induced in healthy subjects, is not reproduced in the critically ill.

\section{REFERENCES}

1. Severinghaus JW. J Appl Physiol Respir Environ Exerc Physiol 1979;46:599.

2. Dash RK, Kroman B, Baasingthwaighte JB. Eur J Appl Physiol 2016;116:97.

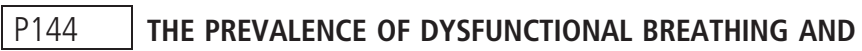 ITS ASSOCIATION WITH PERSONALITY TYPE IN A UNIVERSITY POPULATION}

\section{A Thain, L Silva Vidotto, A Harvey, M Jones. Brunel University, London, UK}

\subsection{6/thoraxjnl-2017-210983.286}

Background Dysfunctional breathing (DB) is an umbrella term used to describe an abnormal breathing pattern which can be psychologically or physiologically based. DB has been shown to be exacerbated at times of increased stress and to be related to anxiety disorders; both factors are common within a university setting, particularly around exam time. Personality types, specifically type A personality, share common risk factors with $\mathrm{DB}$, suggesting a possible association. The prevalence of DB within a university population has not been previously investigated.

Aims To investigate the prevalence of dysfunctional breathing within a university population and assess any association between $\mathrm{DB}$ and type A personality.

Methods A cross sectional study was undertaken involving participants recruited at Brunel University. The primary outcome measure was the Nijmegen questionnaire (validated diagnostic tool for DB), and the secondary outcome measure was the breath hold test (BHT) (clinical diagnostic tool for DB). Additionally, the Behaviour Pattern Scale was used to classify participants as type A or type B personality.

Results 40 participants completed the study. $17.5 \%(7 / 40)$ were positive for $\mathrm{DB}$ on the Nijmegen questionnaire $(\geq 23 /$ 64). Positive scores only occurred in women; consistent with previous data on gender and DB. 7.5\% (3/40) had a positive result using the BHT (<20 s). 50\% of participants were type $\mathrm{A}$ and $50 \%$ type B personality. Pearson's Chi-Square test was used which demonstrated a significant association between DB (Nijmegen questionnaire) and type A personality $(p=0.037)$. No association was found between the Results of the BHT and personality type $(p=0.548)$, or between the Nijmegen questionnaire and BHT Results $(p=0.453)$. At baseline there were no significant differences in participant characteristics, other than gender, between the groups that received a positive or negative DB diagnosis.

Conclusion Dysfunctional breathing may affect a significant percentage of people in a university population; and a significant association with type A personality type has been shown. Raising awareness of DB in the university population may lead to earlier diagnosis and timely referral to physiotherapy or counselling services as appropriate. A larger study is needed to further validate these findings. 